\title{
Preliminary Study on Furniture Innovative Design Based on the Technical Contradiction Matrix of TRIZ
}

\author{
Wenying DONG ${ }^{1,2, a}$, Xiangdong DAI ${ }^{1, b}$,Jiamin $\mathrm{HUANG}^{2, \mathrm{c}}$ You ZHOU ${ }^{2, \mathrm{~d}}$ \\ ${ }^{1}$ Central South University of Forestry and Technology, Changsha, China \\ ${ }^{2}$ Forestry College, Sichuan Agricultural University, Chengdu, China \\ adoreen0656@126.com, b42344070@qq.com, 'c474752253@qq.com, '296759014@qq.com
}

Keywords: TRIZ; Technical contradiction matrix; Furniture; Innovative design

Abstract. To improve the innovative design system of Chinese furniture constantly, the article introduced advanced foreign theory of the solution of inventive problems (TRIZ), and discussed the feasibility and the main way to apply the theory to the innovative design of furniture, which provide a theoretical basis for the research on the innovative method of furniture design based on TRIZ theory later.

\section{Introduction}

It was put forward by Premier Keqiang $\mathrm{Li}$ in < Conference Report of Government Work in $2015>$ that China should accelerate the speed from manufacturing power to create power. In that context, Chinese furniture industry is facing the huge domestic market and strong demand in the international market. To maintain the sustainable development of Chinese furniture industry, in addition to the innovation in management, materials, equipment, technology and other area of the work done, the innovative design is the core of enhancing competitiveness in the market.

China is a big country of furniture production and manufacturing. A mounting number of designers are studying on the innovative design of furniture and regional furniture. Although many companies and research centers put effort on the practice of original design in recent years, and there have been a batch of successful and influential furniture designers and furniture manufacturing enterprises who had the successful practice in traditional culture and innovative furniture. Such as Xiaojie Zhu, LAND BOND and so on. But since the middle of nineteenth Century, western countries began to explore the design of modern furniture, and in 1950s, they had developed into a deep, mature and complete modern furniture system ${ }^{[1]}$. Therefore, compared with the five countries including Italy, Germany and northern European, China has still a lot of question of furniture products about lack of innovation, features, low design and others to be solved.

\section{TRIZ theory and the application}

TRIZ is the abbreviation of" teorija rezhenija izobreta-telskihzadach"in Russian ${ }^{[1]}$, and the English translation is Theory of the Solution of Inventive Problems. This theoretical system is based on the analysis of 2.5 million pieces of high level patents and the comprehensive research on the principles and rules of the multidisciplinary field by the former Soviet inventor Genrikh S. Altshuller ${ }^{[2]}$. With its good maneuverability, systematicness and practicality, it occupies an important position in the field of innovation and creativity in the whole world so that it is considered as a kind of world-class innovative method ${ }^{[3]}$. The main content of TRIZ theory includes two parts: one is the basic system of TRIZ theory; the two is the tool system of solving the problem of TRIZ. The main idea of solving the problem with TRIZ theory is described as follows: as for a specific problem, if we can't find the corresponding solution directly, then, the problem should be transformed and expressed as a problem in TRIZ, so we can obtain the general solution with the method and tools of TRIZ system. Finally, transform the general solution of TRIZ into the solution of specific problems, and realize it in practical problems, then we can get the solution to the problem ${ }^{[4]}$. 
The research of TRIZ theory can be traced back to 1926, but the spread of the theory in China is just more than ten years, and most of the research are used in patent design and mechanical products design; The application in the field of construction is limited in building materials, wall or the optimization of engineering design, or the part of the building structure, materials and measure the degree of damaged ancient architecture using "substance - field model". The research on the application of TRIZ in the enterprise started late. In the 20th century, there was almost no theoretical paper about TRIZ published in China, but since 2008, the innovative methods have been carried out for eight years, the work has made significant achievements. At present, the innovative method pilot demonstration enterprise has reached more than 310, mainly involving machinery, electronics, energy, agricultural machinery, textile and other fields.

\section{The correlation between TRIZ theory and innovative design of furniture}

TRIZ theory system is very large. So far the case applying TRIZ theory to the furniture design are numbered, furthermore, the research on the theoretical system of furniture design based on TRIZ is rare. We need to do that application research mainly aims at the technical contradiction matrix in TRIZ theory, although some of the engineering parameters are not entirely suitable for furniture design, what we found is the same as the argument of Altshuller that the innovative design of the furniture is similar with other innovative design of industry in essence. Applying TRIZ theory to furniture design has strong universality of use, for it not only provides a new train of thought for furniture design, and establishes a new parameter system of furniture design and the innovation principle based on the TRIZ theory, thus provides more methods for the innovative solution of obstacle, but also can broaden the application field of TRIZ theory, and enrich the content system of furniture design.

\section{The key issues of applying TRIZ theory to furniture design}

The 39 engineering parameters. TRIZ theory defined the generally engineering parameters and the expression of practical problems. Altshuller summed up the 39 general engineering parameters to express systemic performance in engineering field including the field of geometry, physical and technical performance. These generally engineering parameters establish the connection between the specific problems and TRIZ theory. In accordance with the Contradiction, when some engineering parameters are improved, it is bound to lead to the deterioration of one or more of the other aspects. Therefore, the parameters of the improvement and the parameters of the deterioration form the technical contradiction, and both the opposites of unity mutually restrain and dependent on each other. In accordance with the analysis of the Altshuller, it can produce about 1300 pairs of typical technical contradictions, almost covers the majority of technical content in projects, pairwise matching the 39 general engineering parameters. When solving practical problem with TRIZ theory tools--technical contradiction matrix, select two of the parameters 39 engineering parameters corresponding to the actual problem to describe the technical contradiction of the system, that is to say, we should transform the contradictions which exists in the actual engineering design to standardized technical contradictions. It means using the engineering parameter in TRIZ theory to describe the actual technical problems. With the organic combination of contradiction and idealism, realize the standardized process of the theory of the solution of inventive problems, which lays the foundation for the standardization of the innovative theory.

The technical contradiction is also usually appears in furniture design, thus before applying TRIZ theory to furniture design, we should make the practical problems of design be standardized. For example, the design of a chair, in the era of personalization, we'd like to have a unique shape of the chair to meet the personalized needs of the users, so designers often design new in order to be different in appearance. Increasing the visual sense of the parameters of improvement, often faced with the problem of reducing the overall structural stability, resulting in the parameters of deterioration of reducing its manufacturing accuracy. The two constitutes a contradictory combination of furniture design. 
The 40 innovative principles. In the process of manufacturing, people are facing the same problems in invention and innovation repeatedly in different periods and fields, and use the same innovative principles to solve the problems repeatedly. Without exception, all the artificial things follow the general law. It can be seen that the solution to the problem (that is, to make the innovation) is regular. There are ways to follow. If we sum up these methods in accordance with the objective law, then they must be applied to all other aspects of the innovative process. To this end, Altshuller analyzed and summarized a large number of invention patents, and extracted 40 inventive principles in TRIZ which have the most practical and universal significance. The 40 inventive principles set up the way to solve the problems and make the invention, so that the method of invention can follow rules, and it makes a breakthrough to solve the practical problems that people thought cannot be solved, even make the void ideal become wealthy. Later, through the analysis and research, it proved repeatedly that the 40 pieces of innovative principles which are most commonly used are the effective ways to solve the problems of technical contradiction.

The technical contradiction matrix. In engineering design, technical contradiction is opposite and related, that is to say if we strengthen some useful functions or reduce some disadvantageous functions, it will produce the complication of deterioration in the other subsystem or the whole system, and the traditional way to solve the problems of technical contradiction is to make a compromise, however, each parameter cannot achieve optimal value, so the solution is not the optimal solution. While TRIZ is trying to make a breakthrough to find the ways to eliminate contradictions, and its goal is to solve the problem by eliminating contradictions, and get a more thorough solution, so that the two sides of the contradiction can achieve a win-win situation, in other words, we can make the two sides of the contradiction -- the two general engineering parameters achieve the best value ${ }^{[5]}$.

To solve the technical problems, the general solution is to first describe the specific practical unresolved issues as a pair of technical contradictions in the 39 general engineering parameters in order to get the standardized model of problem. And then use the TRIZ theory tool--contradiction matrix in view of the different models to find the corresponding innovative principles, so that we can get inspiration from the innovative principle recommended, finally combine with the practical

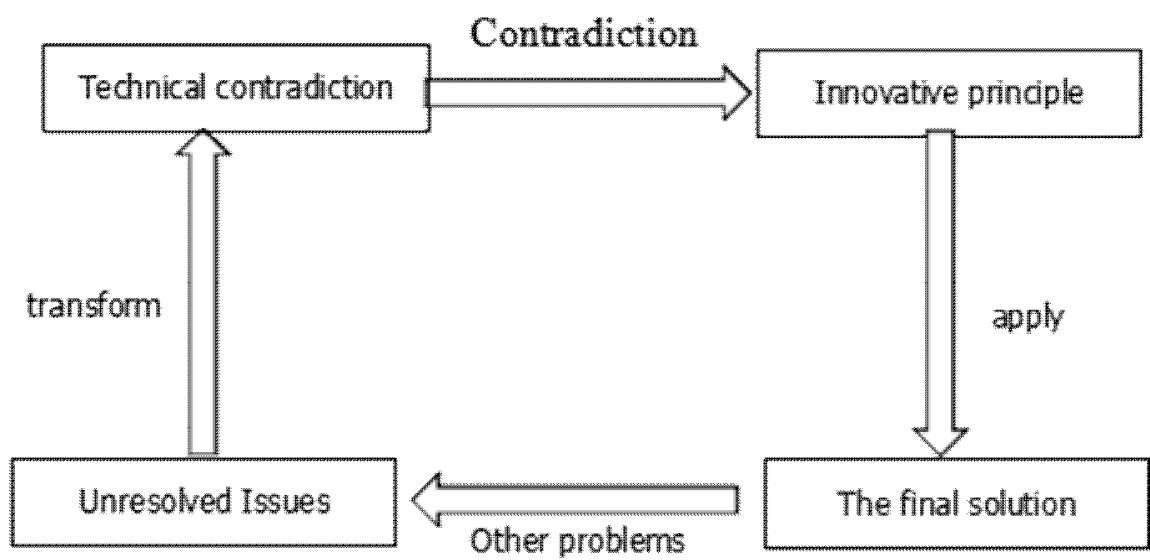
difficulties, and puts forward feasible scheme to solve practical problems. (As shown in figure 1)

\section{An example of innovative design of furniture based on TRIZ theory}

The vast majority of the 39 engineering parameters and the 40 innovative principles of the TRIZ theory are applicable to the field of furniture design, and can guide the optimal design. Such as the design of stored furniture -- wardrobe, obviously, the main requirement of function is more stored space, while ignoring the conditions of the cost, increasing the stored space as an improvement of the parameters, which means that the volume of the wardrobe is expanded, while the indoor space is bound to dwindle. As for the standard of a good furniture, the design of imperfect and unreasonable functional of the wardrobe is the parameter of improvement, so these two constitutes the contradictory combination of furniture design. On the basis of the reference to the 39 engineering parameters table, describe the

\footnotetext{
* From :Liangshen Wang, Fenghua Sun. etc. The innovation theory and application principle of TRIZ. [M]. Science press, 2010
} 
'expand the volume of the wardrobe ' as 'the volume of a moving object' which is the 7th parameters of improvement of the 39 engineering parameters, and then describe the' unreasonable occupation of the wardrobe in indoor space' as' disadvantageous factors generated by the object' which is the 31th parameters of deterioration of the 39 engineering parameters. According to the technical contradiction matrix table of Altshuller, we can find the answers of innovative principles to solve the contradiction is: 17.2.40.1.These four numbers correspond to the principles of "the change of spatial dimension', 'extraction', 'composite' and 'segmentation". It recommended four solutions for the difficulty of the design of the wardrobe, and then according to the sub item of each solution, combining with the actual problem, put forward an effective solution. Such as the "segmentation", the concrete implementation method are 3: to divide an object into separate parts; to divide an object into parts which are easy to assemble and disassemble; to improve the separability of objects. Therefore, the design of the wardrobe can be considered as a form with modular combination, and mainly divided the wardrobe into two types of storage and commonly used modules. Each module can be moved independently and seems to be harmonious and unified, so that when using of the wardrobe, the users can put each module of the wardrobe according to the specific circumstances of room, which saves space. (As shown in figure 2)

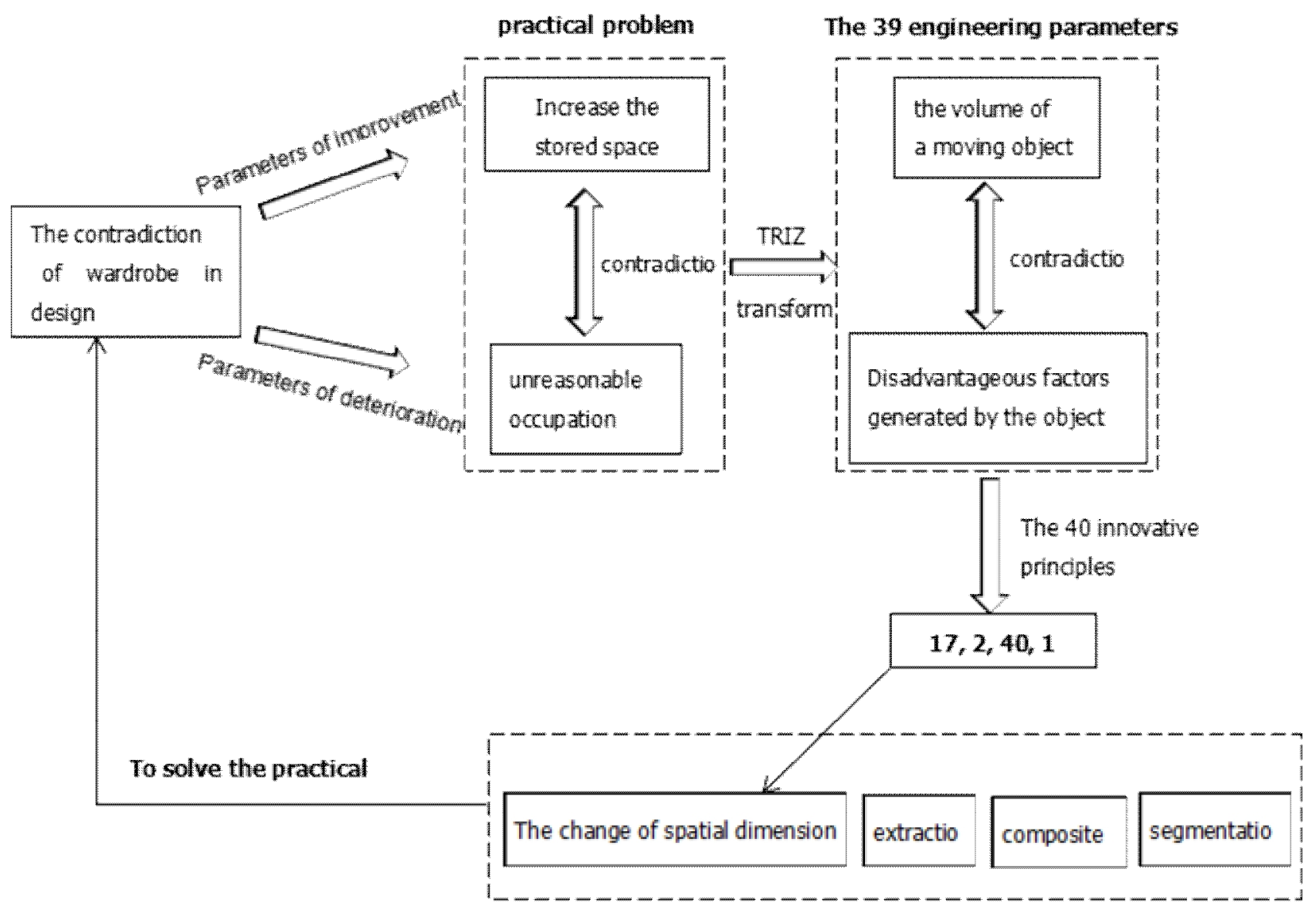

Fig2. An example of innovative design of fumiture based on TRIZ theory

\section{Conclusion}

Since the glorious era of the Ming and Qing furniture, Chines furniture has not formed its own characteristics. After the reform and opening, the idea of advanced design from foreign countries constantly entered in China, and it has prevailed for a time that people blindly worship foreign things ${ }^{[5]}$. In this case, how to integrate the Chinese characteristics with the world, which requires continuous exploration and innovation in furniture design. Designers should not be limited to the current thinking in the field of design, but should departure from the multidisciplinary, multi-disciplinary and 
multi-angle. Reference reasonably and draw lessons from excellent and innovative design theory both at home and abroad, and combine with Chinese excellent traditional culture and regional characteristics, so as to enrich the contents and methods of furniture design, and enhance the influence and competitiveness of Chinese innovative design of furniture. Applying the TRIZ theory to the research of the innovative design in furniture is a new research on the application of foreign TRIZ theory, and it's also a new attempt and application of Chinese traditional regional culture. It pioneered a new paradigm that applies TRIZ theory to the innovative design in furniture.

\section{References:}

[1] RANTANEN K , DOMB E . simplified TRIZ : New problem solving applications for engineers and manufacturing professionals[M] . New York : Auerbach Publishing , 2008

[2] Yunfei Shao, Mao Ye,Xiaowo Tang. The development and solution research of technological innovation method $[\mathrm{J}]$. Journal of Social Science Edition of University of electronic science and technology , 2009(5): : 1-8

[3 ]Runhua Tan. TRIZ and application- - The methods and process of technological innovation[M]. Higher education press, 2010

[4]Liangshen Wang, Fenghua Sun. etc. The innovation theory and application principle of TRIZ. [M]. Science press, 2010

[5] Na Li. Chinese traditional pattern and modern decorative art design [M]. Tianjin: baihua literature and art publishing house, 2011. 